\title{
AUTOMATING TRUST ASSESSMENT FOR CONFIGURATION OF TEMPORARY PARTNERSHIPS
}

\author{
Simon Samwel Msanjila, Hamideh Afsarmanesh \\ University of Amsterdam, (msanjila, hamideh)@science.uva.nl
}

\begin{abstract}
Owing to advances in digital technology, emerging breakthrough solutions responding to market needs are becoming increasingly innovative and complex. Large amount of resources, competencies, skills, etc. are required to accomplish such solutions, usually beyond the capabilities and capacities of a single organization. The emergence of co-innovation networks as breeding environments for creation of temporary partnerships has enhanced the possibility of success for organizations and facilitating their collaborations. One obstacle to configuration of such partnerships as well as collaboration has been the difficulty in assessing the trust level of potential partners. So far, trust level assessment has been performed manually by organizations and in ad hoc manners, which is both time consuming and hardly produces accurate results. Consequently, formation of collaborative initiatives has become more challenging and organizations are reluctant to work with each other. This paper addresses the supervised automation of the assessment of trust level of organizations in co-innovation networks. It presents the design and development of Trust Management system, addressing its specifications and architectures to facilitate its implementation.
\end{abstract}

\section{INTRODUCTION}

Establishing trust relationships among organizations has proved to enhance the efficiency of collaborative processes (e.g. collaborative designing of a new product, etc.) which involve a number of organizations. Organizations are now encouraged to trust others in order to jointly address the need for sharing large amount of resources, competencies, skills, etc. required to respond to a larger opportunity, which none of them could do alone. However, finding trustworthy partners for such collaborations is becoming more challenging.

Traditionally, trust is seen as subjective aspect and thus its evaluation has been opinion-based. Applying subjective based trust analysis, such as opinion-based trust, in collaboration is too risky due to the fact that it can be biased and difficult to formally reason. Approaches for rational trust analysis are needed. We define trust of an organization, as it is applied in VBEs, as the objective-specific confidence of a trustor organization to a trustee organization based on the results of fact-based assessment of trust level of the trustee (Msanjila and Afsarmanesh, 2007c). Thus objective based trust creation refers to the process of creating trust among organizations based on the results of the fact-based assessment of their trust levels. Only measurable or numeric data are applied to the assessment and the resulted trust levels can be supported with some formal reasoning applied during the assessment of trust level, which in turn enhances the reasoning of the established trust relationships. 
A priori to jointly addressing market/society opportunities organizations must both trust each other and prepare themselves for sharing and exchanging the required resources and information, establishing necessary infrastructure for co-working, etc. To address the need of preparing themselves and increase their chances of collaboration, organizations join co-innovation networks that have emerged as breeding environments for the formation of temporary partnerships, such as virtual organizations (VOs). One popular form of co-innovation networks is the VO Breeding Environment (VBE). A VBE is defined as an alliance of organizations and related supporting institutions, adhering to a base long term cooperation agreement, and adopting common operating principles and infrastructures, with the main goal of increasing both their chances and preparedness towards collaboration in potential VOs (Afsarmanesh, et al 2007). VBEs support the preparation of organizations by: (1) maintaining common and sharing principles, (2) providing interoperable infrastructure, (3) facilitating common understanding with a maintained ontology, (4) providing definition of value systems and performance metrics, (5) supporting the assessment of trust level of organizations, etc.

In practice, organizations have been manually assessing trust level of each other both in ad hoc manners which are inefficient, hardly accurate and time consuming. Furthermore, to match the need of quickly responding to opportunities, processes related to assessment of trust level of organizations must now be (semi-) automated. This paper presents an approach and a system for supervised automation of Trust Management to assist the administration of co-innovation networks and specifically VBEs with handling such processes. We define the Trust Management (TrustMan) system which aggregates our previous introduced models and approaches appeared in (Msanjila and Afsarmanesh, 2007 a \& 2007b), and automates the processes related to the management of trust among organizations in VBEs.

\subsection{Assessing trust level of organizations in co-innovation networks}

Perceptions of trust have been in line with the nature of purposes for its applications as well as involved actors. Thus purposes for establishing trust differ among the practices. For each specific practice in which a particular group of actors is involved trust is differently interpreted and perceived as compared to others. In our research, we classify trust aspects into five perspectives: Technological (Tech), Social (Soc), Structural (Str), Managerial (Man), and Economical (Eco) (Msanjila and Afsarmanesh 2007c). Furthermore, to address variation of trust perceptions, a rational approach is required for measuring trust level and reason on the results.

To "rationally" assess trust level of organizations a series of fact-based trust criteria can be applied. With the empirical study of co-innovation networks as well as survey of the past research, we have identified a good number of measurable criteria that act as indicators of trust assessment (Msanjila and Afsarmanesh 2007c). However, we have also identified that the influence of a trust criterion on the trust level can be either positive or negative depending on its behaviour. Furthermore, the behaviour of each trust criterion changes in time, and causally influences others. Causal influences can be studied by applying concepts from system dynamics (Kirkwood, 1998). Results can be translated into mathematical equations reflecting inter-relations of trust criteria (Msanjila and Afsarmanesh 2007c). The formulated equations comprise the base for our designed mechanisms for assessment of trust level of organizations (Msanjila and Afsarmanesh 2007a). Basically, mechanisms developed for TrustMan implement three forms of equations. The $1^{\text {st }}$ form of equation is applied to calculate the final comparative scores of trust level of organizations as an average of weighted scores of all perspectives (equation 1). In all equations the following are applied: TL (trust level), $\mathbf{S}$ (score), per (trust perspective), IF (intermediate factor), W (weight), and Avg (average). 


$$
T L=\operatorname{Avg}\left[\left(W_{T e c h} * S_{T e c h}\right),\left(W_{S o c} * S_{S o c}\right),\left(W_{S t r} * S_{S t r}\right),\left(W_{\text {Man }} * S_{M a n}\right),\left(W_{E c o} * S_{E c o}\right)\right] \ldots
$$

The score for each trust perspective is calculated as weighted average of score for all intermediate factors as shown in equation (2) which represents the $2^{\text {nd }}$ form.

$$
S_{p e r}=\frac{1}{n} \sum_{i}^{n} W_{I F_{i}} * S_{I F_{i}}
$$

The score for intermediate factors is calculated as a function of trust criteria and known factors as shown in equation (3) which represents the third form.

$$
\left.S_{I F}=f \text { 【rust_criteria, known_factors }\right] \quad \text { Where } 0<W_{i}<1 \text {, and } \sum_{\forall i} W_{i}=1
$$

\section{SPECIFICATION OF TRUSTMAN SYSTEM}

The system supports five main kinds of user (Table 1) and based on their requirements the

\begin{tabular}{|c|c|}
\hline $\begin{array}{l}\text { User 1: } \\
\text { VBE } \\
\text { administrat } \\
\text { or }\end{array}$ & $\begin{array}{l}\text { This user group has the highest administrative rights. They can view, and execute all the functionalities } \\
\text { in the system. The requirements for this user include system's support to: (1) Assesses trust level of } \\
\text { membership applicant and VBE members, (2) Define and provide rights to other users, (3) Support other } \\
\text { users to evaluate trustworthiness of trustees (3) Manage trust related data, and (4) Update trust criteria. }\end{array}$ \\
\hline $\begin{array}{l}\text { User 2: VO } \\
\text { planners }\end{array}$ & $\begin{array}{l}\text { This user group gets temporary administration right to perform administrative tasks using. The } \\
\text { fundamental requirements for this user group include system's support to: (1) View the trust criteria } \\
\text { applied to the system, (2) Select specific trust criteria, and (3) Evaluate specific trustworthiness. }\end{array}$ \\
\hline $\begin{array}{l}\text { User 1: } \\
\text { VBE } \\
\text { members }\end{array}$ & $\begin{array}{l}\text { The users in this group possess non-administrative rights and thus they can only access the system to } \\
\text { manipulate their own trust related data. Their requirements include support to: (1) Accesses its base trust } \\
\text { level records, (2) Updates its trust related data, (3) View the trust criteria applied to the system. }\end{array}$ \\
\hline $\begin{array}{l}\text { User 3: } \\
\text { Membershi } \\
\text { p applicants }\end{array}$ & $\begin{array}{l}\text { This user group possesses the very basic rights to support itself with interactions related to its } \\
\text { application submission. The requirement for this user group is to get support provided to submit the trust } \\
\text { related data for the assessment of base trust level. }\end{array}$ \\
\hline $\begin{array}{l}\text { User 4: Ext. } \\
\text { stakeholder }\end{array}$ & $\begin{array}{l}\text { This user group includes customers, and invited organizations. Requirements for this group are } \mathrm{s} \\
\text { support: (1) Customers to create trust to the VBE, and (2) Invited organizations to trust the VBE. }\end{array}$ \\
\hline
\end{tabular}
related functionalities are specified as addressed in section 2.1 .

Table 1: Specification of users and their requirements for TrustMan system

\subsection{Specification of functionalities}

TrustMan system implementation adopted the web services technology. It provides web

\begin{tabular}{|c|c|}
\hline$S 1$ & $\begin{array}{l}\text { For assessing base trust level of organizations: When customizing the TrustMan system, in a specific } \\
\text { environment, the VBE administrator selects the minimum set of trust criteria - base trust criteria - that reflects the } \\
\text { characteristics of the specific domain of the VBE and suits the needs of the environment. The results of the } \\
\text { assessment that apply the base trust criteria are referred to as "base trust level". The service for assessing base } \\
\text { trust level supports the assessment of the trust level of organizations based on the base trust criteria. }\end{array}$ \\
\hline$S 2$ & $\begin{array}{l}\text { For evaluating specific trustworthiness of organizations: This service aims at measuring how trustworthy an } \\
\text { organization is for a specific trust objective, i.e. inviting a VBE member to participate in a VO, etc. The trustor } \\
\text { selects a specific set of trust criteria and defines ratings for values of trust criteria to classify different levels of } \\
\text { trust. TrustMan system applies the selected trust criteria and ratings to evaluate the organizations' trustworthiness. }\end{array}$ \\
\hline S3 & $\begin{array}{l}\text { For establishing trust relationships among organizations: The approach suggested to facilitate establishing trust } \\
\text { relationships among organizations is through measuring their historical data for both their trust records. To create } \\
\text { trust among organizations, they can be provided with relevant information queried from the data stored in the } \\
\text { TrustMan system, which will enable them trusting others. The challenge for the information provision is related to } \\
\text { the four questions of: "who", "when", "why" and "how" (Msanjila and Afsarmanesh, 2007d). }\end{array}$ \\
\hline$S 4$ & $\begin{array}{l}\text { For creating trust to the VBE: This service supports external stakeholders (invited organizations, and customers) } \\
\text { to create trust to the VBE. They need to access information that will assist them with trusting the VBE in relation } \\
\text { to their businesses. This service guides them to access information which fits their trust purposes and perceptions. }\end{array}$ \\
\hline S5 & $\begin{array}{l}\text { For managing trust related data: This service supports VBE: membership applicants, members, and } \\
\text { administrator. Applicants will use this service to submit trust related data to facilitate the assessment of base trust } \\
\text { level for the evaluation of their application towards joining VBEs. Members will use the service to update trust } \\
\text { related data. Administrator will use the service to manage the trust related data, i.e. ensuring its updated, valid, etc }\end{array}$ \\
\hline S6 & $\begin{array}{l}\text { For managing the assessment mechanisms: The equations applied for the development of mechanisms (section } \\
\text { 1.1) for assessing trust level of organizations incorporates weights for the applied parameters. The weights can be } \\
\text { changed from time to time when it is needed using this services. The service is accessed by the administrator only. }\end{array}$ \\
\hline
\end{tabular}
functionalities for human user and web services for system users (Table 2).

Table 2: Specification of functionalities provided by TrustMan system 


\section{ARCHITECTURAL DESIGN OF TRUSTMAN SYSTEM}

Based on the specifications above, we present the architectural design of TrustMan system addressing the operational architecture and the componential architecture.

\subsection{Operational architecture}

TrustMan system is one of the subsystems that together constitute the so-called VBE management system (VMS). The VMS is designed to assists the VBE administration with semi-automatically handling of managerial tasks (Afsarmanesh, et al 2007). TrustMan system assist the VBE administration with handling the tasks related to managing trust among organizations in the VBE. In order to properly and comprehensively provide the required services the TrustMan system interacts with others sub-systems (Figure 1) for four purposes, namely for: (1) acquiring the trust related data, (2) providing results of the trust level assessment, (3) accessing ICT-Infrastructure (ICT-I) basic services, and (4) supporting human access.

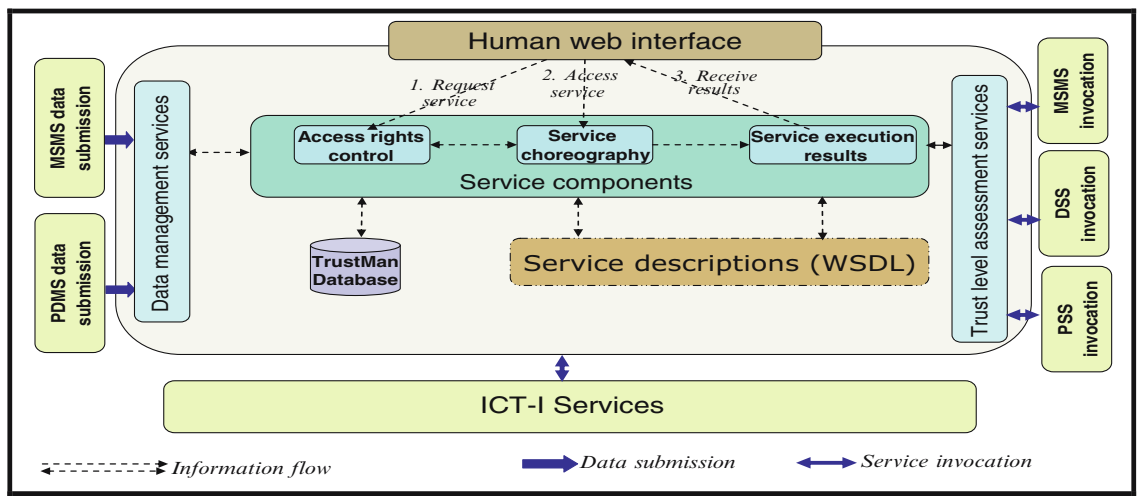

Figure 1. Operational architecture for TrustMan system

(a) Interactions for assisting the acquisition of trust related data

Two sub-systems namely: Membership Structure Management System (MSMS) and Performance Data related Management System (PDMS) interact with TrustMan for submitting trust related data. MSMS is developed to assist the VBE administration with handling the registration of new VBE members, and defining their roles and rights. One fundamental information necessary for the VBE administration to decide about acceptance of the VBE membership applicant is the base trust level. The MSMS interacts with TrustMan to facilitate applicants submit trust related data to the TrustMan system. The interactions are based on service invocations.

While the VBE is in operation phase its members participate in activities, both within the VBE and in configured VOs. Thus their trust related data must be continuously updated. The main source of trust related data is the organizations' performances achieved in those activities. The PDMS interacts with TrustMan to assist the VBE actors with updating the trust related data based on the collected organizational performance. The PDMS includes management systems for VO information, inheritance, and VBE's activities related performance. 


\section{(b) Interactions related to accessing results for trust level assessment}

Some VMS subsystems need to invoke a number of services provided by TrustMan system to access the records of trust level of organizations. The trust levels of organizations in this case are used as input towards providing the required services by those subsystems to their respective users. Three VMS subsystems are identified which need to invoke services provided by TrustMan, namely: MSMS, Decision Support System (DSS), and Partner Search and Suggestion (PSS) (Figure 1).

MSMS shall invoke the service for assessing base trust level of membership applicant for the aim of analyzing whether the applicant organization meets the specified minimum level of trust in the VBE. Thus the MSMS uses the resulted trust level and other relevant information to provide services which support the VBE administrator on deciding about acceptance of the application.

DSS supports the VBE administration to make decisions on a number of issues but mainly in relation to controlling and alarming member organizations for: the $V B E$ competency gap, the organization's lack of performance, and the organization's low trustworthiness. Specifically for this case, in order to analyze the evolution of trust level of the organization the DSS shall invoke the services provided by TrustMan system for assessing base trust level of organizations. The interactions take place in scheduled manner and thus the organizations whose trust level is deteriorating can be alerted and advised on enhancing their trustworthiness.

PSS assists the VO planner with selecting suitable VO partners among the VBE members. One key activity during the selection of suitable VO partners is the evaluation of their specific trustworthiness. The PSS interacts with the TrustMan to facilitate the VO planer with evaluating the trustworthiness of VO partners.

(c) Interactions to access the ICT Infrastructure (ICT-I) basic services

The TrustMan system needs to invoke some basic services provided by the ICT-I to effectively provide required services. There are a number of basic services provided by the ICT-I developed in the ECOLEAD project (www.ecolead.org). TrustMan needs to invoke two basic services from the ICT-I, namely the service for: data access, and security management (Rabelo, et al 2006). The service for data access supports the TrustMan system with handling trust related data in its database, such as the related interactions with MSMS and PDMS for data acquisition. The service for security management supports the TrustMan system to authenticate remote users.

\section{(d) Interactions related to the human access of the TrustMan system}

The interactions between human users and the TrustMan system are facilitated and achieved through the web interface. In addition to providing web services which are invoked by other remote systems, such as MSMS, DSS, PSS, etc., TrustMan system provides functionalities which can be accessed by human users on the web interface.

\subsection{Componential architecture}

Componential architecture of TrustMan system adopts the standard definitions of web service technology and specifically addressing the classification of layers. 
The presentation layer (Figure 1) handles the delivery of information from the process layer to the web interface in a format that is readable by humans. The layer also handles the transformation of data submitted by human user to the format that is acceptable by various modules at the process layer. As such, it relieves the process layer of concerns regarding syntactical differences in data representation which is understandable by the human end-user based on the web format (Field and Hoffner, 2003). The presentation layer is the only layer where people can care about what they are sending at an advanced level than a bunch of ones or zeros (Rhody, 2002).

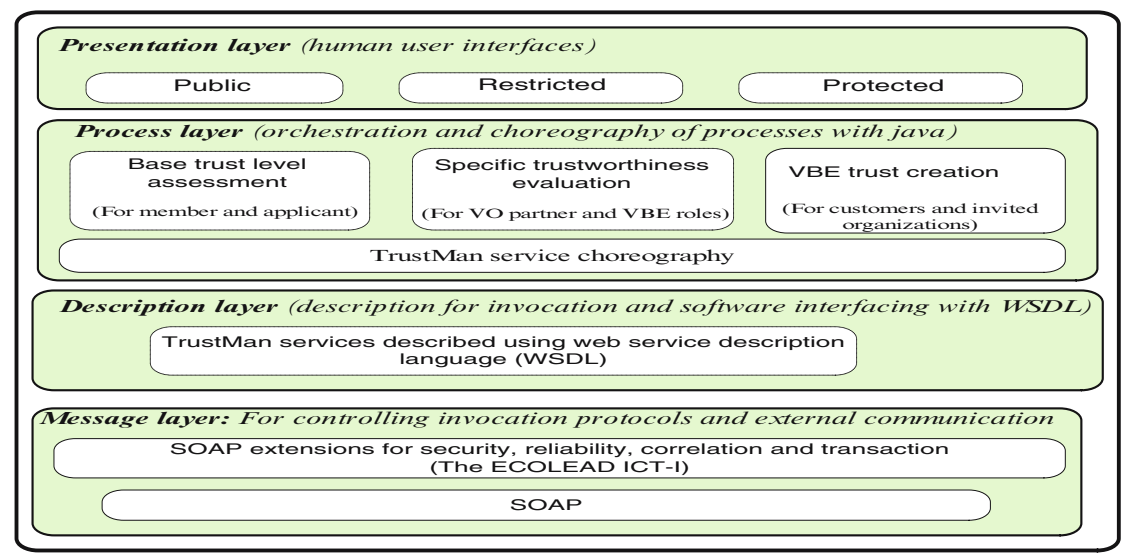

Figure 2. Componential architecture for TrustMan system

TrustMan manages, and deals with sensitive information which in most cases the VBE members consider as confidential such as strategic business data. In TrustMan system, at this layer, the web interface that facilitates the accessibility of information as well as the execution of various supported functionalities applies user rights as addressed in "service 3 " in section 2.1, namely: public, restricted and protected interfaces. Modules for public interface belong to the group of result provisional components in the operational architecture. Modules for restricted and protected interfaces constitute components that belong to both components for "access right control" (user rights and roles) and components for "service execution results" (presenting records) in the operational architecture as shown in Figure 1.

\section{(b) Layer 2: Process layer}

In daily life, activities are scheduled in a way that each one is known when it will be performed; following which activity, and which activity will follow soon after the preceding activity is completed. Similarly, when a service is invoked, processes that must be executed shall be organized. The process layer is responsible for defining the logic of execution of various processes (modules) to provide the requested service. The process scheduling constitutes of orchestration and choreography.

Orchestration refers to the logic (the sequence and flow) of execution of functions within one system process (Papazoglou and Georgakopoulus, 2003). For example, in java programming this refers to the logic of the execution of functions within one object. Figure 3 shows orchestration of several processes integrated in one choreography process, e.g. system control, trustworthiness evaluation, etc.

Choreography represents the logic that will be followed to execute various modules including invoking other services in order to provide a single integrated service (Peltz, 
2003). Several web services were choreographed to provide required integrated services. To exemplify in this paper, we present the choreography of an integrated service for evaluating specific trustworthiness of organizations. Figure 3 represents the choreography of a set of services constituting the evaluation trustworthiness and thus represents a partial processes' architecture of the TrustMan.

The process layer is the only layer which constitutes the services that are scheduled and executed by users and thus all components in this layer belong to the group of components for "service choreography" in the operational architecture.

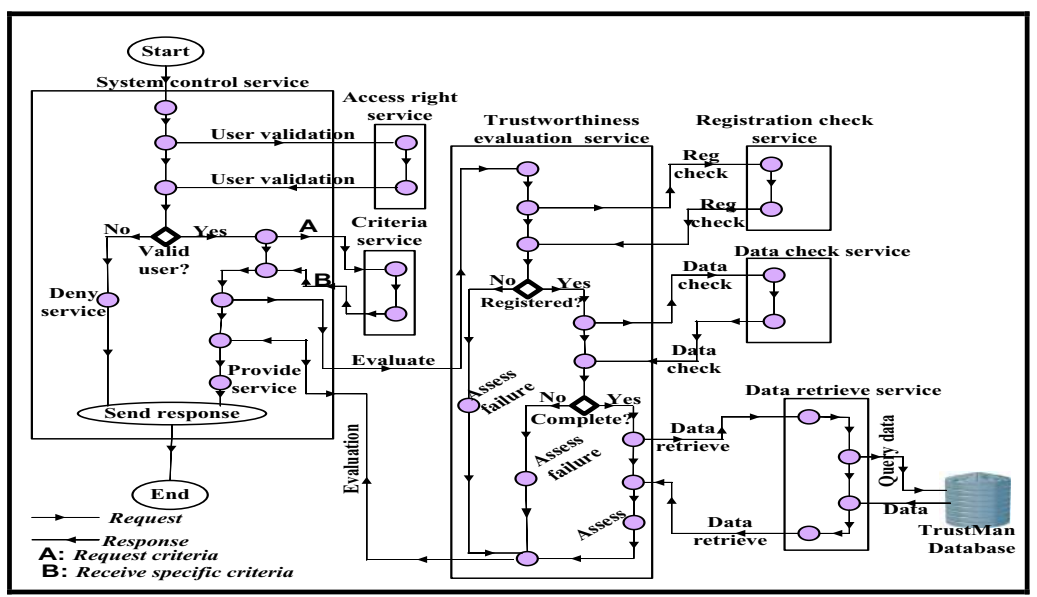

Figure 3. Choreography of a service for evaluating specific trustworthiness of organizations

\section{(c) Layer 3: Description layer}

The description layer handles the provision of grammatical specifications of services available at a certain site. From definition as applied in web service technologies the description of a service applies WSDL (web service description language). WSDL describes four fundamental parts of the service. The first part is Public interface, which describes the public operations that are visible to external partners. The second part is data type information for all message related to requests and responses that describe the data types for the variables that should be passed to access each service. The third part is binding information related to the transport protocol, which defines the protocols necessary to access the service and facilitate external communication. Lastly is the address information for locating the specified service, which describes the server location, and how it is discovered in the UDDI.

WSDL files for java web services are generated from the respective java classes. A number of WSDL files describing the services provided by TrustMan system were generated. This layer represents similar aspects as the service description part in the operational architecture as shown in Figure 1.

\section{(d) Layer 4: Message layer}

Message layer defines the protocols for communication, credential information, and it sends that information across the network so that a receiving server/client can be able to interpret it (Peltz, 2003). The standard communication protocol for web services is SOAP (Simple Object Access Protocol). On top of the standard SOAP protocol, addition mechanisms can be added to enhance: security, reliability, adaptability, etc. The 
ECOLEAD project has developed the so-called ICT infrastructure (ICT-I) for providing the necessary measures to enhance interactions among services for supporting the collaborations in the VBE (Rabelo. et al 2006).

\section{IMPLEMENTATION OF TRUSTMAN SYSTEM}

Main functionalities provided by TrustMan system are developed using javaprogramming language. A number of classes were implemented in different "java project packages" which were logically classified based on the global view of the system's modules. Figure 4 shows modules of TrustMan system and each module constitutes a number of classes as presented below addressing their purposes.

Module for trust level assessment: This module constitutes classes which provide basic algorithms for assessing trust level of an organization. It constitutes classes which implements generic algorithms for assessing organization's trust level.

Module for static assessment: The classes in this module extend the classes included in the module for trust level assessment to reuse the implemented basic algorithms. The classes implemented in this module provide algorithms to assess trust level of an organization based on static set of trust criteria for all perspective. In this case the trust criteria to be applied to all cases are known and fixed.

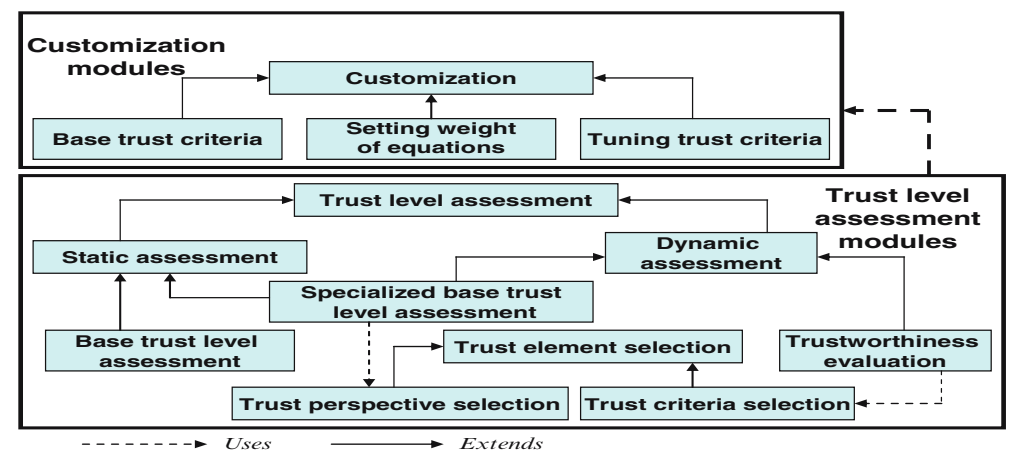

Figure 4. Global view of modules for TrustMan system

Module for base trust level assessment: The classes in this module extend the classes included in the module for static assessment to reuse the implemented algorithms. The assessment of base trust level applies the set of trust criteria selected by the VBE administrator a priori to the VBE establishment. Thus these trust criteria are known to the system. In this module additional classes are implemented to support the rating and generalization of trust level of an organization.

Module for dynamic assessment: The classes in this module extend the classes included in the module for trust level assessment to reuse the implemented basic algorithms. The classes implemented in this module provide algorithms to assess trust level of an organization based on dynamic set of trust criteria. In this case the trust criteria to be applied for the assessment are selected by the user. Thus dynamic assistance for selecting and applying the trust criteria for the assessment of trust level of organizations is implemented in this module.

Module for trust element selection: This module constitutes classes which provide algorithms for selecting trust elements for supporting dynamic trust level assessment. The classes in this module are inherited by classes in two other modules, namely: (1) Module 
for trust perspective selection which supports the dynamic selection of trust perspective, and (2) Module for trust criteria selection supporting dynamic selection of trust criteria.

Module for trustworthiness evaluation: The classes in this module extend the classes included in the module for dynamic assessment. The classes in this module constitute algorithms for evaluating specific trustworthiness of an organization. Also, this module uses the classes in the module for trust criteria selection.

Module for specialized base trust level assessment: The classes in this module extend classes in both modules for dynamic and static assessment. The classes in this module extend the classes in the module for dynamic assessment to reuse the algorithm for supporting the assessment of trust level of organizations adapting the dynamic selection of trust perspectives. When trust perspective is known all the trust criteria becomes static and thus classes implementing static algorithms are inherited. This module uses the classes in the module for trust perspective selection.

\section{ADAPTABILITY AND REPLICABILITY OF TRUSTMAN}

In our approach for assessing the trust level of organizations a large comprehensive set of trust criteria is defined and applied. Each VBE however may apply a different set of "base trust criteria" which constitutes those criteria that are selected by the VBE administrator, during the customization stage of TrustMan system. The selection of base trust criteria depends on the preferences and perceptions of trust perspective of both "VBE administrator and VBE members". Furthermore, as in daily life, these preferences and perceptions may also change with time. To handle such situations, TrustMan system must be replicable and easily adaptable to support easy customization such as the following modules of TrustMan system (Figure 4).

- Module for customizing trust criteria: The mechanisms for assessing trust level of organizations are designed based on mathematical equations (section 1.1). The adaptation and replication of TrustMan system is enhanced by facilitating the possibility to change the set of base trust criteria, without modifying the implemented mechanisms. Thus all general trust criteria are supported in the system, but only the selected set of base trust criteria are applied in assessing organizations' trust level. "Logical operations" are implemented in the module to ease the change of constituents of the set of base trust criteria for each VBE.

- Module for customizing the weights of elements in the equations: As described in section 1.1 the implemented mathematical equations consists of weights for each applied parameter, such as each trust criteria. The setting of these weights also depends on the domain and specific preferences of the VBE environment. This module supports the VBE administrator to modify the weights of equations.

- Mechanisms for supporting tuning of trust criteria: The set of trust criteria applied in the design of mechanisms for assessing trust level of organizations is identified in collaboration with the VBE networks participated in ECOLEAD project. Since the set is generalized avoiding the specificity of different networks, the identified trust criteria may need to be tuned for VBEs when some conditions changes, such as the emergence of new trust objectives. The TrustMan provides mechanisms for defining new trust criteria its tuning. 


\section{CONCLUSION}

This paper addresses the automation of processes related to the assessment of trust level of organizations in VBEs. It contributes to both the formalization and architectural development of a formal trust management system. The presented system applies formal mechanisms, which are based on mathematical equations for assessing the trust level of organizations. Thus the presented mechanisms can be used to support rational reasoning about the assessed trust level of organizations. Furthermore, the system is designed and implemented based on web services technology, and therefore its adaptability, replicability and sustainability are also technologically supported. Moreover, the modules developed for supporting the customization of TrustMan system in different VBE environments, enhance the ease of its adaptability and replicability.

\subsection{Acknowledgement}

This work was supported in part by the ECOLEAD project funded by the European Commission. The authors thank for contributions from partners in the ECOLEAD consortium.

\section{REFERENCES}

1. Afsarmanesh, H., Camarinha-Matos, L., Msanjila, S.S. "Virtual organizations breeding environments: key results from ECOLEAD". In the proceedings of International conference on Cost Effective Automation in Networked Product Development and Manufacturing, 2007.

2. Field, S., Hoffner, Y. Web services and matchmaking. International journal of networking and virtual organizations, Inderscience 2003; Vol. 2, No. 1: 16-32.

2. Kirkwood, C. W. System Dynamics Method. Ventana System Inc. 1998.

3. Msanjila, S.S., Afsarmanesh, H. Trust Analysis and Assessment in Virtual Organizations Breeding Environments. International Journal of Production Research, ISBN (print) 0020-7543, Research, Taylor \& Francis, 2007a, pg. 1-43

4. Msanjila, S.S., Afsarmanesh, H. Modeling trust relationships in Collaborative Networked Organizations. International Journal of Technology Transfer and Commercialization, ISBN (print): 1470-6075, Inderscience, 2007b, Vol. 6, No. 1, pg. 40-55.

5. Msanjila, S.S., Afsarmanesh, H. "HICI: An approach for identifying trust elements - The case of technological perspective in VBEs". In proceeding of International conference on availability, reliability and security (ARES-2007), Vienna, 2007c, pg. 757-764.

6. Msanjila, S.S., Afsarmanesh, H. "Specification of the TrustMan system for assisting management of VBEs". In the lecture notes of computer science series, LNCS 4657, Springer, 2007d, pg 34-43.

7. Papazoglou, M.P., Georgakopoulus, D. Service-Oriented Computing. Communications of the ACM 2003; Vol 46, No. 10.

8. Peltz, C. Web services orchestration and choreography. IEEE computer 2003; Vol. 36, No. 10.

9. Rabelo, R.J., Gusmeroli, S., Arana, C., Nagellen, C. "The ECOLEAD ICT infrastructure for collaborative networked organizations”. In: Camarinha-Matos, L., Afsarmanesh, H., Ollus, M. (eds.) IFIP International Federation for Information Processing. Network-Centric Collaboration and Supporting Frameworks, 2006, vol. 224, pp. 161-172.

10. Rhody, S. Why web services. Web services journal, 2002; vol. 2, issue 2. 